$2^{\text {nd }}$ Dept. of Internal Medicine, Iuliu Hatieganu University of Medicine and Pharmacy, Cluj-Napoca, Romania

\title{
Lactobacillus reuteri versus triple therapy for the eradication of Helicobacter pylori in functional dyspepsia
}

Iulia Antonia Pop Muresan, Lucian Liviu Pop, Dan L. Dumitrascu

\begin{abstract}
Background and aim. The eradication of $H$. pylori infection using PPI associated with different combinations of two or three antibiotics entails high risks of side effects and non- adherence. Therefore probiotics have been proposed for $H$. pylori eradication.

We tested the efficacy of Lactobacillus reuteri plus Pantoprazole compared to a triple regimen based on Pantoprazole plus Amoxicillin plus Clarithromycin in patients with $H$. pylori infection and functional dyspepsia.
\end{abstract}

Methods. In a prospective design, 46 patients (M: 13, F: 33, mean age $48.80 \pm 13.82$ years) fulfilled the following inclusion criteria: age at least 18, documented informed consent, positive $H$. pylori finding by histology, no morphological changes of the gastric mucosa at upper gastrointestinal endoscopy and complaints of functional dyspepsia according to the Rome III criteria. Exclusion criteria were: presence of any other chronic organic diseases that required drug treatment, use of antibiotics, PPIs or $\mathrm{H} 2$ antagonists in the previous 3 months; pregnancy or lactation. Patients were randomly divided into two equal groups (23 patients each group). One group received the standard therapy in our area: Pantoprazole $40 \mathrm{mg}$ bid for 30 days associated with Amoxicillin 2x1000 mg/day and Clarithromycin $500 \mathrm{mg}$ bid for 14 days. The other group received Pantoprazole $40 \mathrm{mg}$ /day plus L. reuteri DSMZ 17648 twice a day for 8 weeks. Post-treatment eradication was tested by $H$. pylori antigen stool assay at 30 days after therapy.

Results. The group on L. reuteri plus Pantoprazole presented $65.22 \%$ eradication rate compared to $73.91 \%$ cure rate in the group that received the Pantoprazole and Amoxicillin and Clarithromycin therapy, with no statistically significant difference in eradication rate between the two groups $(\mathrm{p}=0.75)$. The total adherence was good and eradication of $H$. pylori was associated with improvement of dyspeptic symptoms for both eradication regimens.

Conclusion. L. reuteri is a good alternative for patients with chronic dyspepsia for the eradication of $H$. pylori infection. Its efficacy is similar to the triple therapy.

Keywords: dyspeptic symptoms, Helicobacter pylori, Lactobacillus reuteri, probiotics

\section{Introduction}

Helicobacter pylori (H. pylori) is a gram negative, spiral-shaped, microaerophilic human pathogen that colonizes the gastric mucosa of more than $50 \%$ of the global population [1,2]. H. pylori is able to survive the acid environment of the stomach due to its ability to adhere to the gastric mucosa, colonizing the mucosal lining of the stomach [3]. Most H. pylori carriers remain asymptomatic; however, it is associated with a number of gastrointestinal diseases such as peptic ulcer $(10-20 \%)$, gastric cancer (1-2\%) and rarely mucosa-associated lymphoid tissue lymphoma [4]. There is evidence of an association between the amount of gastric colonization by $H$. pylori and the chance of developing symptoms of disease [5]. In the National Health and Nutrition Examination Survey III, H. pylori was strongly positively associated to gastric cancer mortality [6]. The $5^{\text {th }}$ edition of the Maastricht Consensus Report offers diagnostic guidelines and recommends always therapeutic regimes for $H$. pylori eradication [7].

H. pylori is encountered in $30 \%$ to $50 \%$ of functional dyspeptic patients, but 
its pathogenic implications are not yet established [8,9]. In dyspeptic patients a test-and-treat strategy is suggested. Therapeutic options for $H$. pylori infection with the purpose of eradication include different combinations of proton pump inhibitors associated with two or three antibiotics. Unfortunately this complex approach has high risks of side effects and non-compliance [10].

Probiotics are used in the treatment of $H$. pylori infection [11]. They have proven helpful in reducing side effects of antibiotics and enhance patient compliance [12]. Strains of Lactobacillus reuteri ( $L$. reuteri) have been shown to exert an inhibitory effect on the settlement of human gastric mucosa by H. pylori [13]. L. reuteri has the capacity to produce reuterin, a wide spectrum antibiotic active against $H$. pylori [14]. L. reuteri strain DSMZ 17648 was tested for antibiotic resistance and no resistance was found [5].

The aim of this study was to test the efficacy of $L$. reuteri in eradication of $\mathrm{H}$. pylori in an area where data on antibiotic resistance are very scarce.

\section{Methods}

\section{Subjects}

The study population included recently diagnosed $H$. pylori positive subjects referred to a tertiary gastroenterology unit by family physicians or specialists from other medical centers, for chronic dyspeptic symptoms.

Subjects were included in this study if they had reached the age of 18 , had documented informed consent, a positive $H$. pylori finding, had no morphological changes of the gastric mucosa in upper gastrointestinal endoscopy and presented of functional dyspepsia (FD) according to the Rome III criteria [15].

Patients were excluded if they had any other chronic organic diseases that required drug treatment: myocardial ischemia or infarction, congestive heart failure, diabetes mellitus, renal failure, cirrhosis, previous gastric surgery, gallstone disease, chronic pancreatitis, chronic obstructive pulmonary disease, rheumatoid arthritis or regular use of nonsteroid anti-inflammatory drugs or bisphosphonates, oral intake of antibiotics, PPIs or H2 antagonists in the precedent 3 months, pregnancy or lactation.

\section{Protocol}

Baseline H. pylori infection was defined as the presence of $H$. pylori on histological examination of gastric biopsies.

At baseline, patients were evaluated for symptoms, history of allergy to any of the drugs used, and absence of prior treatment for $H$. pylori infection. Demographic data including age, gender, height and weight were collected for each patient. Body mass index was also calculated. We investigated severity of five symptoms (general abdominal symptoms, satiety, fullness, bloating and epigastric pain) by using independent visual analogue scale (VAS) (0-100 $\mathrm{mm}$ ) and the anxiety score by using Hamilton's Anxiety
Scale. The measurements were made at baseline and at 30 days after therapy.

We undertook a prospective single center comparative study between $L$. reuteri plus PPI versus the standard of care consisting in triple therapy plus PPI.

Patients were randomly divided in two equal groups. One group received Pantoprazole $40 \mathrm{mg}$ bid. for 30 days associated with Amoxicillin $1000 \mathrm{mg}$ bid and Clarithromycin $500 \mathrm{mg}$ bid (PAC) for 14 days. The other group received Pantoprazole $40 \mathrm{mg}$ /day plus $L$. reuteri DSMZ 17648 (Pylopass, Remedia Laboratories, Romania) twice a day (PLR) for 8 weeks.

All drugs in this study were self-administered as tablets. Adherence was checked at the end when patients were asked to bring the drug packages.

Post-treatment eradication success was defined by the negative $H$. pylori antigen stool assay at 30 days after therapy.

\section{Statistics}

Statistic assessments were performed with a commercially available package and included descriptive statistics and ANOVA comparison between groups. The significance levels between comparisons was set at 0.05 .

\section{Ethical issues}

The study was approved by the local ethical committee and conducted in accordance to the Helsinki regulations on experiments on humans.

\section{Results}

A total of 46 FD patients (M: 13, F: 33); with a mean age of $48.80 \pm 13.82$ years fulfilled inclusion criteria. FD patients were divided into three subgroups according to the cardinal symptoms: Postprandial Distress Syndrome (PDS), Epigastric Pain Syndrome (EPS) and overlapping PDS and EPS $[16,17]$. They were then randomly divided into two equal groups (23 patients each group). The baseline demographic data are given in Table I.

Table I. Baseline demographic data of treated patients.

$\begin{array}{lccc} & \text { L. reuteri } & \text { Triple therapy } \\ \text { No. of patients } & 23 & 23 \\ \text { PDS/EPS/Overlap } & 12 / 7 / 4 & 11 / 8 / 4 \\ \text { Male/Female } & 5 / 18 & 8 / 15 \\ \text { Mean age }(\text { years }) \pm \text { SD } & 50.00 \pm 1.50 & 47.61 \pm 16.25 \\ \text { BMI - mean }(\mathrm{kg} / \mathrm{m} 2) \pm \mathrm{SD} & 25.42 \pm 3.3 & 26.83 \pm 3.09\end{array}$

The first group, receiving PLR therapy, showed $65.22 \%(15 / 23)$ eradication rate. The second group, receiving PAC therapy, showed $73.91 \%$ (17/23) eradication rate. There wass no statistically significant difference in eradication rate between the two groups $(\mathrm{p}=0.75)$. There was no drop out in this study.

The therapy adherence for the entire group was $97.83 \%$. One patient from the PLR group did not fully comply with treatment due to side effects consisting in 
nausea and heartburn, which lead to a compliance rate of $95.65 \%$ in this group. The PAC group had a 100\% rate of compliance. Five patients from this group reported heartburn, nausea and epigastric pain, but all this sideeffects were self-limiting, did not required specific therapy and improved until the end of the treatment.

There was no correlation between BMI and eradication rate.

In both groups, the eradication of $H$. pylori was associated with improvement of dyspeptic symptoms and anxiety score. There was no difference between the two groups regarding the alleviation of symptoms (Table II).

Table II. Symptoms and anxiety score changes in both groups.

\begin{tabular}{l|c|c|c}
$\begin{array}{l}\text { Parameter } \\
\text { (final - initial) }\end{array}$ & $\begin{array}{c}\text { L. reuteri } \\
(\mathbf{n}=\mathbf{1 6})\end{array}$ & $\begin{array}{c}\text { Triple therapy } \\
(\mathbf{n}=\mathbf{1 7})\end{array}$ & Value of $p$ \\
$\begin{array}{l}\text { General } \\
\text { symptoms }\end{array}$ & $6.50 \pm 1.21$ & $6.82 \pm 0.88$ & 0.192 \\
Satiety & $5.31 \pm 1.14$ & $5.06 \pm 0.90$ & 0.241 \\
Fullness & $5.25 \pm 0.86$ & $4.65 \pm 0.93$ & 0.031 \\
Bloating & $5.06 \pm 1.24$ & $4.71 \pm 0.69$ & 0.155 \\
Pain & $2.75 \pm 1.06$ & $2.94 \pm 0.75$ & 0.276 \\
Anxiety & $2.50 \pm 0.63$ & $2.65 \pm 0.70$ & 0.266
\end{tabular}

\section{Discussion}

Despite the fact that the $H$. pylori infection is recognized as an etiological factor in gastrointestinal pathology including gastric cancer, only a part of the patients actually develop symptoms [18]. Eradication is recommended as the standard procedure for patients who develop acute symptoms, and it should also be performed in the infected population. Unfortunately, in the general population this desiderate may be not accomplished, especially in regions with high prevalence of $H$. pylori infection, high rate of antimicrobial resistance or poorly developed health systems [5].

There have been many attempts to develop an effective treatment protocol to eradicate $H$. pylori infection. For decades, the use of antimicrobials was the first choice of therapy. The most effective treatment regimens are quite complicated, requiring the ingestion of three or four different drugs at multiple intervals of time and for a longer time [19]. Borody et al where the first to develop a therapy with a high eradication rate in $H$. pylori infection. It involved three drugs: bismuth, metronidazole and tetracycline administrated for 10 to 14 days without a PPI [20]. Association of a PPI and increased dose of metronidazole improved eradication rates [21]. The triple therapy approach, PPI and amoxicillin and clarithromycin, provided low eradication rates in some regions, explained by the pretreatment antibiotic-resistant strains [22]. The choice of antibiotics requires the knowledge on microbial antibiotic resistance in each specific area. Unfortunately, in our country there are no data on $H$. pylori antibiotic resistance or sensitivity. Therefore, using probiotics for $H$. pylori eradications seems to be attractive.

Indeed, in the last decades the efficacy of probiotics for $H$. pylori eradication was tested to avoid antimicrobial resistance, allergies or other antibiotic side effects. $L$. reuteri DSMZ 17648 showed a significant decrease of H. pylori stomach colonization in vivo study [5]. A pilot study conducted by Dore et al showed that $L$. reuteri have potential role in $H$. pylori eradication. L. reuteri plus pantoprazole bid was able to eradicate $13.6 \%$ of patients with $H$. pylori infection [23].

Starting from this hypothesis, we tested efficacy of L. reuteri plus pantoprazole compared with pantoprazole plus amoxicillin plus clarithromycin in our area (Western Romania). Our results show a good eradication rate in the L. reuteri plus pantoprazole group (65.22\%) much higher than the one showed by Dore et al. [23]. This can be explained by the geographical differences which involve different cultural and alimentary habits.

In the pantoprazole and amoxicillin and clarithromycin group we also had a good $H$. pylori eradication rate, and this is similar to other studies involving this therapy [24].

The only drawback seems to be the long time administration period, which might lead to lack of compliance in these patients, and the relative high cost for this treatment. Our data are consistent with other data from reviews suggesting that probiotics may be considered as alternative therapy to the antibiotic therapy for $H$. pylori eradication $[25,26]$.

Eradication of $H$. pylori was associated with improvement of dyspeptic symptoms in both groups, but we noticed an increase of these symptoms in the first days of administration of pantoprazole plus amoxicillin plus clarithromycin in some patients. We consider this finding as an explanation for lack of adherence in patients which develop this adverse effect.

Given the fact that data on the association of $H$. pylori infection and BMI are conflicting [27] and some authors found a positive association between $H$. pylori infection and an increased BMI [28], we have analyzed data in our groups which included patients with a mean BMI classified according to the World Health Organization recommendations as overweight $(25-29.9 \mathrm{~kg} / \mathrm{m})$ and found no correlation between BMI and eradication rate.

Our study represents to our knowledge the first attempt in our area to test the efficacy of $L$. reuteri plus PPI in eradicating $H$. pylori.

However, this prospective trial has some limitations. The sample size is small, neverthelss the data are useful and open the hope to an alternative in patients with unknown antibiotic sensitivity, with adverse effects or reluctant to ingest antibiotics. Further trials of larger amplitude and multicentric would be useful to establish the role of the probiotics in eradicating $H$. pylori. 


\section{Conclusions}

L. reuteri in association with pantoprazole eradicates H. pylori. It has a very good compliance and almost no side effects. It is useful in areas with ignored antibiotic resistance to antibiotics for $H$. pylori. Its non-inferiority versus the PAC triple therapy should be confirmed by further trials.

\section{References}

1. Clyne M, Dolan B, Reeves EP. Bacterial factors that mediate colonization of the stomach and virulence of Helicobacter pylori. FEMS Microbiol Lett. 2007;268:135-143.

2. Peleteiro B, Bastos A, Ferro A, Lunet N. Prevalence of Helicobacter pylori infection worldwide: a systematic review of studies with national coverage. Dig Dis Sci. 2014;59:1698-1709.

3. Blaser MJ. Helicobacters are indigenous to the human stomach: duodenal ulceration is due to changes in gastric microecology in the modern era. Gut.1998;43:721-727.

4. Ford AC, Axon AT. Epidemiology of Helicobacter pylori infection and public health implications. Helicobacter. 2010;15 Suppl 1:1-6.

5. Mehling H, Busjahn A. Non-viable Lactobacillus reuteri DSMZ 17648 (Pylopass ${ }^{\mathrm{TM}}$ ) as a new approach to Helicobacter pylori control in humans. Nutrients. 2013;5:3062-3073.

6. Chen Y, Segers, S, Blaser MJ. Association between Helicobacter pylori and mortality in the NHANES III study. Gut. 2013;62:1262-1269.

7. Malfertheiner P, Megraud F, O'Morain CA, Gisbert JP, Kuipers EJ, Axon AT, et al. Management of Helicobacter pylori infection-the Maastricht V/Florence Consensus Report. Gut. 2017;66:6-30.

8. Bytzer P, Talley NJ. Dyspepsia. Ann Intern Med. 2001;134(9 Pt 2):815-822.

9. Talley NJ, Stanghellini V, Heading RC, Koch KL, Malagelada JR, Tytgat GN. Functional gastroduodenal disorders. Gut. 1999;45 Suppl 2:37-42.

10. Ramakrishna BS. Helicobacter pylori infection in India: the case against eradication. Indian J Gastroenterol. 2006;25:2528.

11. Ritchie ML, Romanuk TN. A meta-analysis of probiotic efficacy for gastrointestinal diseases. PLoS One. 2012; 7:e34938.

12. Dore MP, Goni E, Di Mario F. Is there a role for probiotics in Helicobacter pylori therapy? Gastroenterol Clin North Am. 2015;44:565-575.

13. Mukai T, Asasaka T, Sato E, Mori K, Matsumoto M, Ohori H. Inhibition of binding of Helicobacter pylori to the glycolipid receptors by probiotic Lactobacillus reuteri. FEMS Immunol Med Microbiol. 2002;32:105-110.
14. Chung T, Axelsson S, Lindgren SE, Dobrogosz WJ. In vitro studies on reuterin synthesis by Lactobacillus reuteri. Microb Ecol Health Dis. 1989;2:137-144.

15. Drossman DA. Rome III: The functional gastrointestinal disorders, 3rd ed. MacLean, VA: Degnon Associates, Inc., 2006.

16. Talley NJ, Walker MM, Holtmann G. Functional dyspepsia. Curr Opin Gastroenterol. 2016;32:467-473.

17. Talley NJ, Ford AC. Functional Dyspepsia. N Engl J Med. 2015;373:1853-1863.

18. Go MF. Review article: natural history and epidemiology of Helicobacter pylori infection. Aliment Pharmacol Ther. 2002;16 Suppl 1:3-15.

19. Graham DY. Hp-normogram (normo-graham) for assessing the outcome of $\mathrm{H}$. pylori therapy: effect of resistance, duration, and CYP2C19 genotype. Helicobacter. 2016;21:85-90.

20. Borody TJ, Cole P, Noonan S, Morgan A, Lenne J, Hyland L, et al. Recurrence of duodenal ulcer and Campylobacter pylori infection after eradication. Med J Aust. 1989;151:431-435.

21. van der Hulst RW, Keller JJ, Rauws EA, Tytgat GN. Treatment of Helicobacter pylori infection: a review of the world literature. Helicobacter. 1996;1:6-19.

22. Dore MP, Piana A, Carta M, Atzei A, Are BM, Mura I, Massarelli G, Maida A, Sepulveda AR, Graham DY, Realdi G. Amoxycillin resistance is one reason for failure of amoxycillin-omeprazole treatment of Helicobacter pylori infection. Aliment Pharmacol Ther. 1998;12:635-639.

23. Dore MP, Cuccu M, Pes GM, Manca A, Graham DY. Lactobacillus reuteri in the treatment of Helicobacter pylori infection. Intern Emerg Med. 2014;9:649-654.

24. Buzás GM. Quality of life in patients with functional dyspepsia: Short- and long-term effect of Helicobacter pylori eradication with pantoprazole, amoxicillin, and clarithromycin or cisapride therapy: A prospective, parallelgroup study. Curr Ther Res Clin Exp. 2006;67:305-320.

25. Chey WD, Leontiadis GI, Howden CW, Moss SF. ACG Clinical Guideline: Treatment of Helicobacter pylori Infection. Am J Gastroenterol. 2017;112:212-239.

26. Goderska K, Agudo Pena S, AlarconT. Helicobacter pylori treatment: antibiotics or probiotics. Appl Microbiol Biotechnol. 2018;102:1-7.

27. Sotuneh N, Hosseini SR, Shokri-Shirvani J, Bijani A, Ghadimi R. Helicobacter pylori infection and metabolic parameters: is there an association in elderly population? Int J Prev Med. 2014;5:1537-1542.

28. Suki M, Leibovici Weissman Y, Boltin D, Itskoviz D, Tsadok Perets T, Comaneshter D, et al. Helicobacter pylori infection is positively associated with an increased BMI, irrespective of socioeconomic status and other confounders: a cohort study. Eur J Gastroenterol Hepatol. 2018;30:143-148. 